\title{
Burden of carbapenem non-susceptible infections in high-risk patients: systematic literature review and meta-analysis
}

\author{
Esther E. Avendano ${ }^{1 *} \mathbb{D}$, Gowri Raman ${ }^{1,2}$, Jeffrey Chan ${ }^{1}$ and Eilish McCann ${ }^{3}$
}

\begin{abstract}
Background: Owing to their resistance to an important class of antibiotics, the prevention and treatment of carbapenem-resistant (CR)/non-susceptible Gram-negative (GN) infections has become an important public health objective. We conducted a systematic review and meta-analysis of published literature to evaluate the burden of CR GN infections, focusing on high-risk patients such as transplant recipients, or patients with cancer, renal impairment, or sepsis.
\end{abstract}

Methods: MEDLINE ${ }^{\circledR}$, Cochrane Central, and Embase ${ }^{\circledR}$ were searched between 2010 and March 2019. Abstracts and full-text articles were screened in duplicate. Random effects meta-analysis was conducted when reported outcomes were sufficiently similar.

Results: Twenty-six publications were eligible. Meta-analyses found increased mortality associated with CR infections among high-risk patients in both unadjusted analysis (8 studies; summary unadjusted odds ratio [OR]: 5.85; 95\% confidence interval [Cl]: 3.69, 9.26; $\left.\right|^{2}=19.8 \%$ ) and adjusted analysis (5 studies; summary hazard ratio [HR]: 4.67; 95\% Cl: $\left.2.18,9.99 ; 1^{2}=77.7 \%\right)$, compared to patients with carbapenem-susceptible (CS) infections or no infection. Increased mortality was also seen in subgroup analyses by length of follow-up (either short-term or long-term) or causative pathogen. A limited number of studies found that CR GN infections increased the risk for mechanical ventilation, adverse events such as graft failure or acute rejection in solid organ transplant recipients, increased renal failure or nephrotoxicity, and an increase in readmissions and costs, though the findings reported in the literature were not consistent.

Conclusion: This systematic literature review and meta-analysis indicates that CR GN infections in high-risk patients are associated with increased mortality, emphasizing the need for antimicrobial stewardship and infection control in hospitals which treat high-risk patients and for the development of effective antimicrobials with favorable efficacy and safety profiles for the treatment of CR GN infections.

Keywords: Carbapenem-resistance, Gram-negative, High-risk, Mortality

*Correspondence: eavendano@tuftsmedicalcenter.org ${ }^{1}$ Institute for Clinical Research and Health Policy Studies, Center for Clinical Evidence Synthesis, Tufts Medical Center, Boston, MA, USA Full list of author information is available at the end of the article

\begin{abstract}
Introduction
An alarming increase in antibiotic-resistant Gram-negative (GN) infections represents a burden on healthcare systems globally [1-3]. The Centers for Disease Control classifies carbapenem-resistant (CR) Enterobacteriaceae as a major public health threat and the World Health Organization considers CR Pseudomonas aeruginosa as a critical priority pathogen requiring immediate and
\end{abstract} original author(s) and the source, provide a link to the Creative Commons licence, and indicate if changes were made. The images or other third party material in this article are included in the article's Creative Commons licence, unless indicated otherwise in a credit line to the material. If material is not included in the article's Creative Commons licence and your intended use is not permitted by statutory regulation or exceeds the permitted use, you will need to obtain permission directly from the copyright holder. To view a copy of this licence, visit http://creativecommons.org/licenses/by/4.0/. The Creative Commons Public Domain Dedication waiver (http://creativeco mmons.org/publicdomain/zero/1.0/) applies to the data made available in this article, unless otherwise stated in a credit line to the data. 
aggressive action [4]. CR/carbapenem non-susceptible GN pathogens are typically resistant to most of the antibiotics used in routine clinical practice, in addition to the carbapenem class. The increase in CR GN infections has led to a resurgence in the use of older antibiotics i.e., broad antimicrobial therapies such as polymyxins that were seldom utilized in the recent past due to efficacy, dosing, and/or toxicity concerns [5]. As a result of these factors, the prevention and effective treatment of CR GN infections has become an important public health objective.

Certain risk factors increase the likelihood of a patient becoming colonized or infected with GN bacteria. These include exposure to healthcare settings [6-9], use of invasive devices or procedures [10-12], receipt of prior antimicrobial therapy [13], foreign travel [14, 15], and being classified as 'high-risk' [16-18]. To expand upon the latter, 'high-risk' can refer to certain clinical characteristics or comorbidities (e.g., hematological or solid malignancies), receipt of concomitant medication(s), or surgical procedures (e.g., organ transplant). In addition to having a greater risk of acquiring GN pathogens, organ transplant recipients, patients undergoing major surgeries, and immunosuppressed patients are predisposed to poor clinical outcomes including increased morbidity and mortality; this is further compounded by the GN infection $[19,20]$. No recent literature review has examined the burden of CR GN infections (and the burden relative to that of carbapenem-susceptible [CS] GN infections) among these high-risk patients or in a critical care setting where these patients typically receive treatment. Given the lack of comprehensive information on these important patient populations, we propose to conduct a systematic literature review (SLR) evaluating recent studies on the burden of CR GN infections among high-risk patients.

\section{Methods}

We conducted a SLR and meta-analysis of published literature to evaluate the burden of CR GN infections in patients classed as 'high-risk', including but not limited to those receiving treatment for cancer or receiving an organ transplant, those with renal impairment, and severely ill patients such as those with sepsis or septic shock, and in critical care settings e.g., intensive care units (ICU), skilled nursing facilities, long-term care facilities.

\section{Data sources and study eligibility}

A comprehensive search to evaluate contemporary literature was conducted in the MEDLINE ${ }^{\circledR}$, Cochrane Central, and Embase ${ }^{\circledR}$ databases from January 2010 through March 2019 for citations that included terms related to pathogens (e.g., Klebsiella, Pseudomonas, Escherichia), mode of infection (e.g., nosocomial, hospital-acquired), carbapenem resistance (e.g., [carbapenem, imipenem, meropenem] and resistant), treatment setting (e.g., hospital, ICU, intensive, critical, skilled nursing, long-term acute care), and patient characteristics (e.g., transplant, chronic renal insufficiency, malignancy, ventilatordependent, immunocompromised, sepsis, bacteremia, septicemia, septic shock) (Additional Table 1; Additional Table 2].

All citations identified by literature searches were independently screened by at least two researchers using abstrackr [21]. Training sessions were implemented where all researchers screened the same articles until all team members reached consensus on the application of the eligibility criteria. Full-text publications for citations that met the inclusion criteria were retrieved and screened in duplicate. Any disagreements that arose during citation or full-text screening were resolved through discussion.

\section{Study inclusion criteria}

We included various study types and designs (prospective and retrospective, with or without a comparator group) conducted in hospitalized adults ( $\geq 18$ years) infected with CR GN pathogens. If studies included a comparator group, this could comprise patients with a 'CS infection' or with 'no infection' (i.e., patients were colonized). In addition, some studies included a comparator group where patients were confirmed not to have a CR infection, but with no explicit statement as to whether they were colonized or had a CS infection; in these instances the comparator was classified as 'no CR infection'. Studies including patients with healthcare-associated infections (including hospital-acquired/nosocomial infections) in hospitals, nursing facilities, critical care units, or ICUs, in the US, Canada, and Western Europe (economically well-developed European nations including Germany, UK, Italy, France, Spain, Portugal, Belgium, Netherlands, and Luxembourg) were included. Outcomes of interest included all-cause mortality, infection-related mortality, length of stay in hospital or ICU, readmission, mechanical ventilation, adverse events such as nephrotoxicity, and economic outcomes such as direct costs (associated with treatment and length of stay) and total costs.

\section{Study exclusion criteria}

We excluded abstract-only publications, cross-sectional studies, case reports, case series, narrative reviews, and any studies published before 2010. The rationale for excluding studies published prior to 2010 was to keep studies as similar as possible with regards to the definitions of carbapenem resistance and the epidemiology 
of the causative pathogens. We excluded studies that did not provide results for adults, healthcare-associated infections, countries of interest, or CR infections. Studies including patients with $>20 \%$ Gram-positive coinfections, reporting on specific antimicrobial therapies, or not reporting results for at least one of the outcomes of interest were excluded. We also excluded studies that included only colonized patients and did not include separate results for patients with CR infections.

\section{Data extraction}

A customized data extraction form was created in Microsoft Excel $^{\mathrm{TM}}$ to gather relevant data elements from included studies. The data extraction form was tested on several studies and revised before full data extraction. Extracted data included variables addressing study design features, enrolled and analyzed sample sizes, study population characteristics, infection characteristics, description of exposure and comparator groups, relevant outcomes, results (percentages, univariate, multivariate), and factors to inform the risk of bias assessment. Any data missing or unavailable in the publications were deemed 'not reported. Data from each study were extracted independently by one of three investigators and confirmed by at least one other. Any data discrepancies were identified and resolved through discussion.

\section{Analysis}

When necessary, unadjusted odds ratios (OR) were calculated. For each outcome, meta-analyses were conducted when basic criteria were met. For effect size measures (i.e., OR, hazard ratio [HR]), if at least three studies (or independent cohorts) provided sufficient data, standard random effects model metaanalyses were performed capturing the chi-squared $P$ value (values $<0.10$ were deemed to be significant) and the $\mathrm{I}^{2}$ statistic. For the purpose of meta-analysis, all effect size measures (OR, HR, relative risk [RR]) were treated as being equivalent. Subgroup meta-analyses were also performed by length of follow-up (in hospital and $\leq 30$ day follow-up, $>30$ day follow-up), CR pathogen (Klebsiella pneumoniae, $P$. aeruginosa), comparator arm (e.g., CS infection, no infection), and high-risk 'types' (e.g., transplant, chronic renal insufficiency, malignancy). If a study reported results for more than one comparator group then priority in analyses was given to CS, followed by no CR, and then no infection. If a study reported results for both $\leq 30$ day and $>30$ day mortality, then $\leq 30$ day was used in the analyses. All reported comparators and lengths of follow-up were included in the respective subgroup analyses. Studies that were heterogeneous in terms of outcome definitions were not combined in a meta-analysis, but were descriptively summarized as part of this systematic review. All analyses were performed in Stata version 13 (StataCorp, College Station, Texas).

\section{Risk of bias/quality assessment}

Two reviewers independently assessed the risk of bias for each included study. Reviewers used the Agency of Healthcare Research and Quality (AHRQ) Risk of Bias assessment and assessed the following methodological quality items: selection bias, performance bias, detection bias, information bias, and appropriate outcome measurements. Each quality item was rated as Low, High, or Unclear.

\section{Results}

The literature search (Additional Table 1; Additional Table 2) identified 2819 citations, and 159 abstracts were eligible for full-text screening (Fig. 1). Full-text publications were retrieved and 133 publications were excluded during full-text screening (Additional Table 3). A total of 26 publications met the inclusion criteria (Table 1); of these, 12 studies included only high-risk patients: eight studies solely included patients who were solid organ transplant recipients, three only included patients with hematologic malignancies, and one study only included patients who had undergone open-heart surgery. The remaining 14 studies did not solely focus on high-risk patients; 11 included a subset of high-risk patients within the total population, and three included a general population of critical care patients. Twenty-two studies included patients infected with $K$. pneumoniae, two included patients infected with $P$. aeruginosa, and two focused on mixed Enterobacteriaceae infections. Sixteen studies compared at least one outcome of interest in patients with CR infection, with patients that had a CS infection, no CR infection, or no infection, while the remainder only reported results for patients with $C R$ infection (Additional Table 4). Eight studies were prospective observational studies and the remainder were retrospective observational studies. Fourteen studies were conducted in the US, 11 in Italy, and one in Spain. Four studies received government funding, five studies received funding from various entities, eight studies reported not having received any funding, and nine studies did not report a funding source. Most studies were conducted in tertiary teaching/academic hospitals; two studies did not report this information. The sample size across the included studies ranged from 18 patients to 632 patients. The median age of included patients ranged from 51 years to 74 years, and the proportion of male study subjects ranged from 35.7 to $81.0 \%$. 


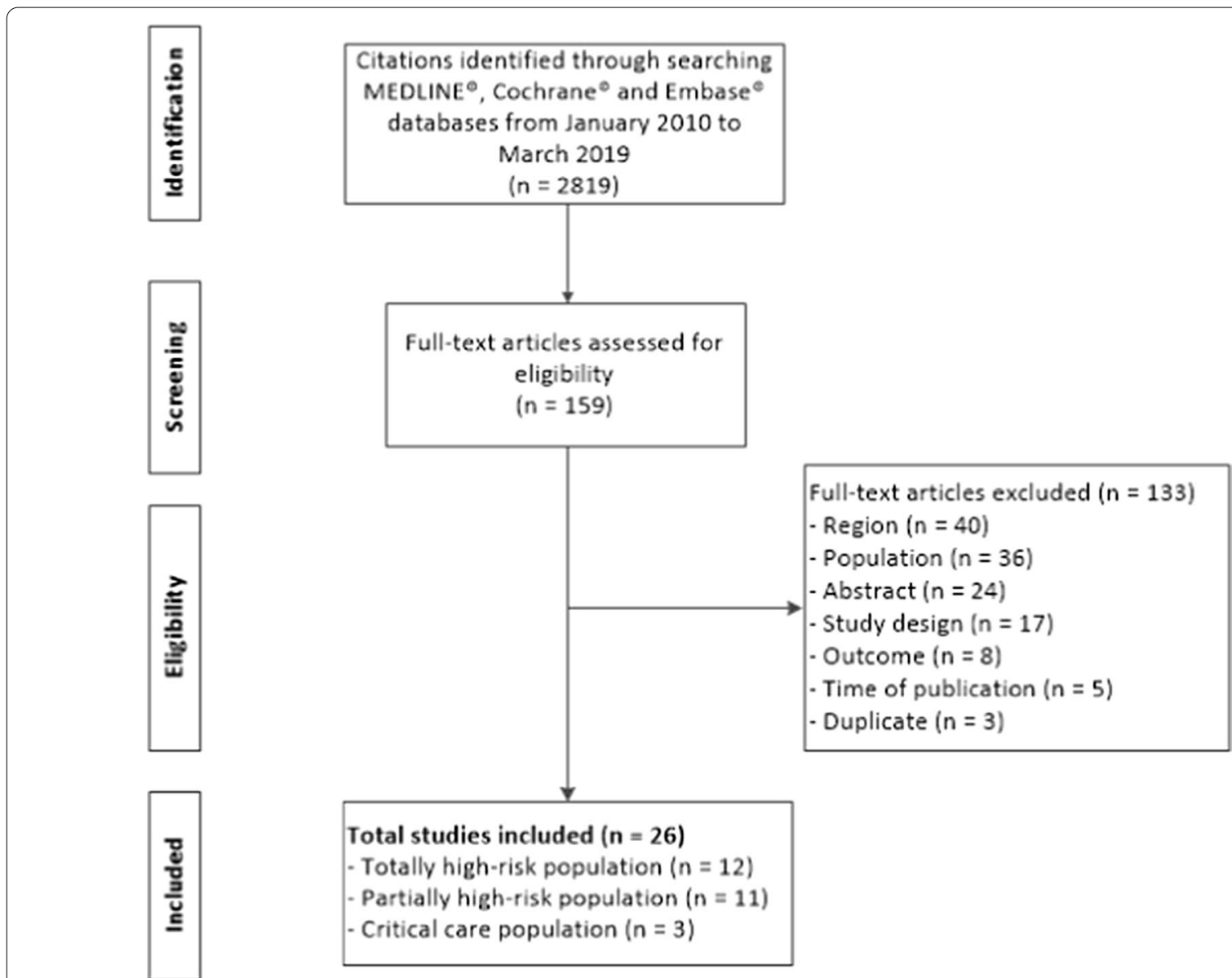

Fig. 1 Study flow diagram. Flow diagram detailing the number of studies included and excluded at each step of the systematic review search and selection process

\section{Mortality}

Twenty-five studies reported mortality in patients with CR infections [13, 22-32, 34-46]. Of these, 12 compared mortality in patients with $C R$ infections with mortality in patients without a CR infection (e.g., CS infection, no CR infection, no infection), which permitted the calculation of unadjusted ORs $[13,22,27-30$, $32,36-38,42,45], 11$ provided results for patients with CR infection only [23-26, 34, 35, 39, 41, 43, 44, 46], and two $[31,40]$ reported 30-day mortality for patients with CR infection only and either 6-month or 1-year mortality for both patients with CR infection and patients with no CR infection. Mortality was reported across pathogen types and type of comparator arm. Twelve studies reported data for high-risk patients exclusively (solid organ transplant, hematologic malignancy, and open-heart surgery patients); of these, eight studies compared mortality in CR infected patients with controls $[13,31,32,37,38,40,42,45]$, and six studies provided results for the $C R$ infection patients only $[24,31$,
34, 40, 41, 46] (two studies reported both comparative and non-comparative data, depending on the outcome).

\section{Meta-analysis of mortality data from studies of totally high-risk patient populations}

The eight studies that focused on high-risk patients only and compared CR infections with controls all provided unadjusted data, and five of these studies also reported adjusted data. Meta-analysis was conducted using the eight studies which reported unadjusted data [13, 31, 32, $37,38,40,42,45]$ and found a significant increase in mortality risk for high-risk patients with CR K. pneumoniae (CRKP) infections $(n=290)$ (summary OR: 5.85 ; 95\% CI: $3.69,9.26 ; \mathrm{I}^{2}=19.8 \%$ ) compared to controls (either patients with CS $K$. pneumoniae [CSKP] or patients without an infection [ $n=1062]$ ) (Fig. 2). In sensitivity analysis, excluding two studies [31, 42] with an unclear risk of bias (Additional Table 6), the analysis still found a significantly increased risk of mortality among patients with CR infections compared to controls (summary OR: 


\begin{tabular}{|c|c|c|c|c|c|c|c|}
\hline 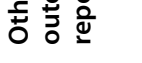 & $\Sigma$ & $\Sigma$ & $\Sigma$ & 岇 & $\Sigma$ & $\Sigma$ & 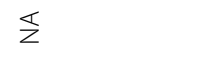 \\
\hline 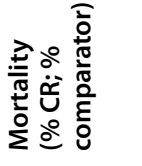 & 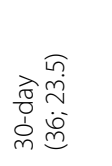 & 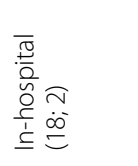 & 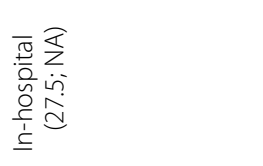 & 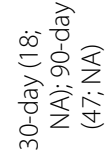 & 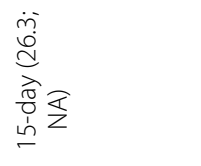 & 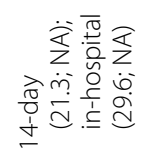 & 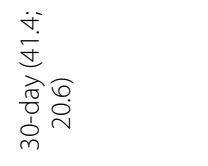 \\
\hline 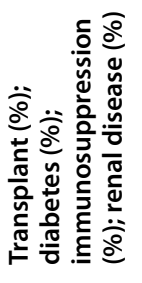 & 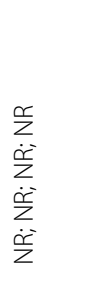 & 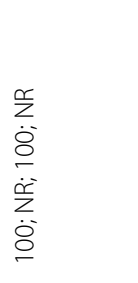 & 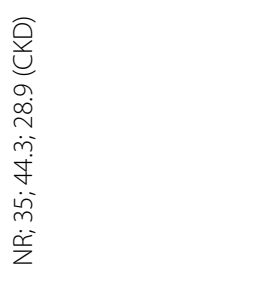 & $\begin{array}{l}\infty \\
\infty \\
\infty \\
\stackrel{0}{0} \\
\stackrel{0}{0} \\
\dot{\hat{\phi}} \\
\dot{0} \\
\dot{0}\end{array}$ & 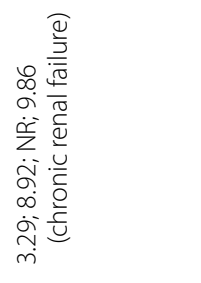 & 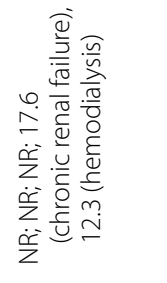 & 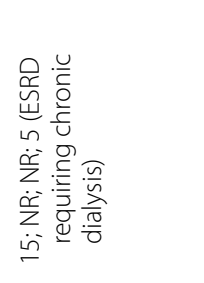 \\
\hline 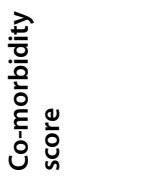 & $\frac{a}{z}$ & $\frac{o}{z}$ & 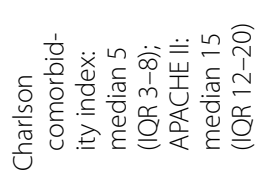 & 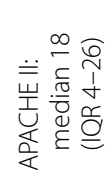 & 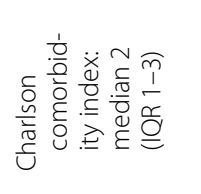 & 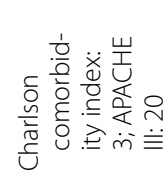 & 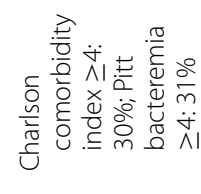 \\
\hline 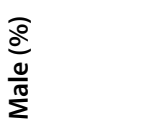 & ֶֻ. & $\stackrel{\text { mे }}{2}$ & $\frac{9}{6}$ & in & 㫄 & $\tilde{6}$ & in \\
\hline 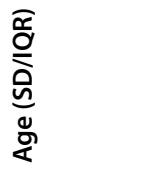 & 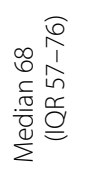 & 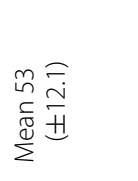 & 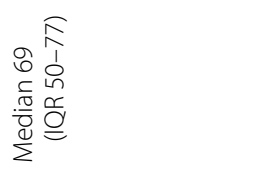 & 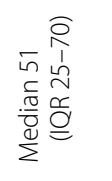 & 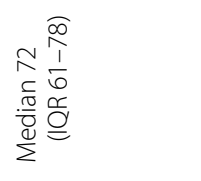 & 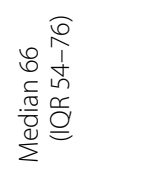 & 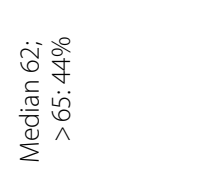 \\
\hline 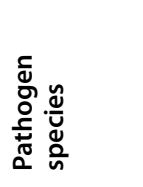 & 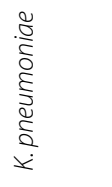 & 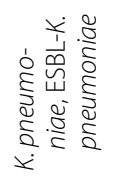 & 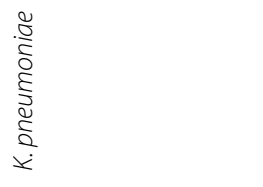 & 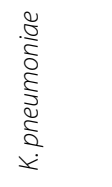 & 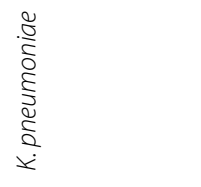 & 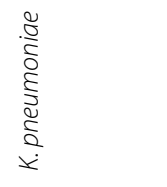 & 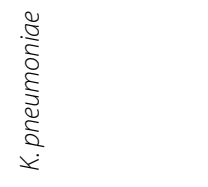 \\
\hline 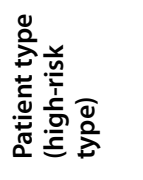 & 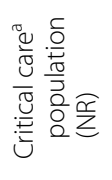 & 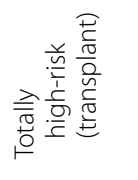 & 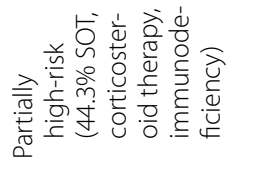 & 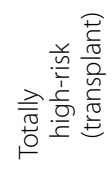 & 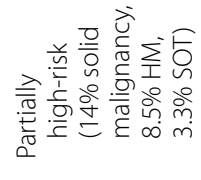 & 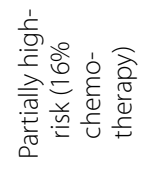 & 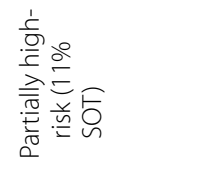 \\
\hline 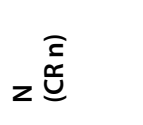 & 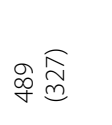 & $\stackrel{\infty}{\circ} \underset{d}{d}$ & $\sigma \widehat{\sigma}$ & $\simeq \stackrel{E}{E}$ & $\begin{array}{l}\underset{\frac{m}{d}}{\bar{m}} \\
\bar{n}\end{array}$ & 孞仝 & 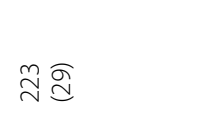 \\
\hline 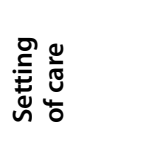 & 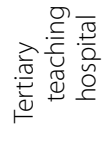 & 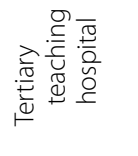 & 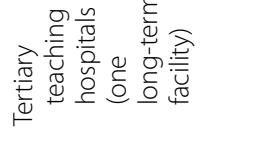 & 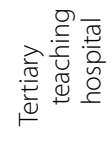 & 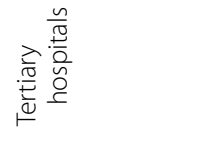 & 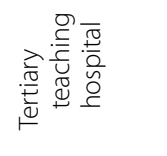 & 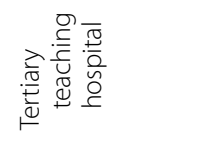 \\
\hline 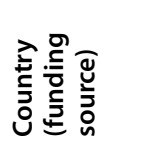 & 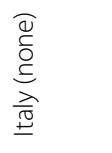 & $\begin{array}{l}\stackrel{\widetilde{c}}{\Sigma} \\
\stackrel{心}{J}\end{array}$ & $\begin{array}{l}\widehat{\partial} \\
\text { o } \\
\text { ते } \\
\text { 馬 }\end{array}$ & 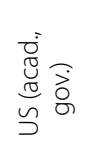 & 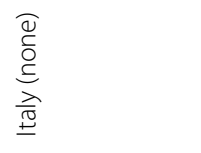 & 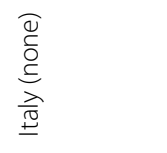 & 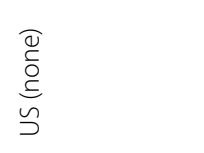 \\
\hline 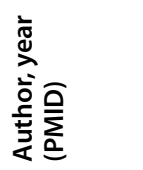 & 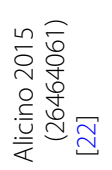 & 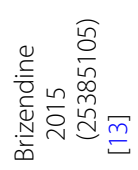 & 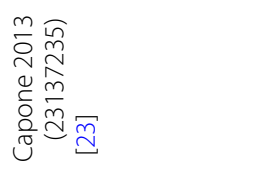 & 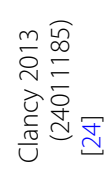 & 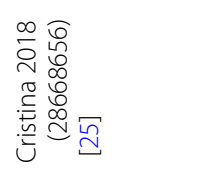 & 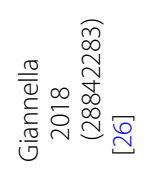 & 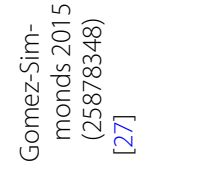 \\
\hline
\end{tabular}




\begin{tabular}{|c|c|c|c|c|c|c|c|}
\hline 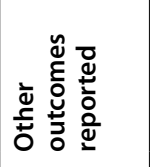 & $\tilde{O}$ & $\Sigma$ & 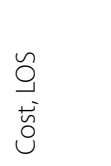 & $\overleftarrow{z}$ & 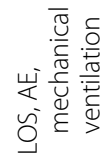 & 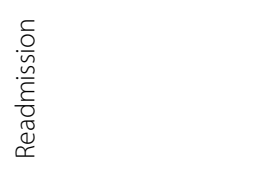 & 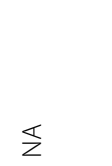 \\
\hline 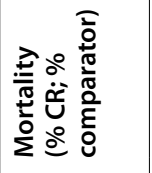 & 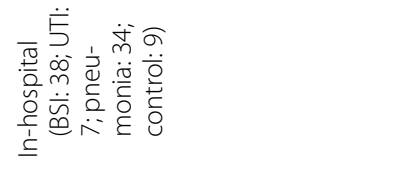 & 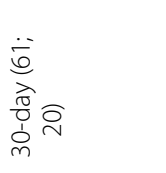 & 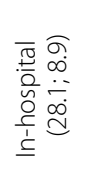 & 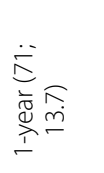 & 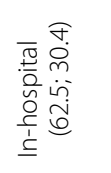 & $\mathbb{z}$ & 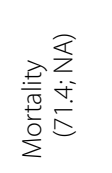 \\
\hline 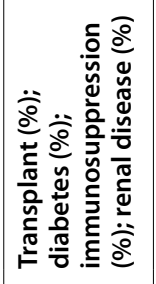 & 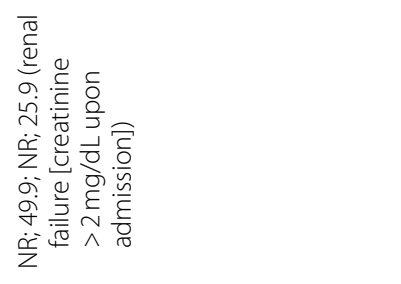 & 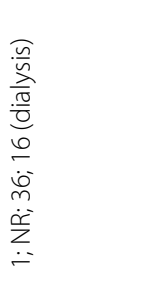 & 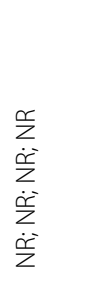 & 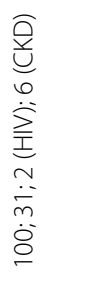 & 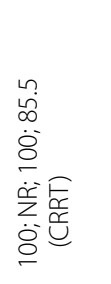 & 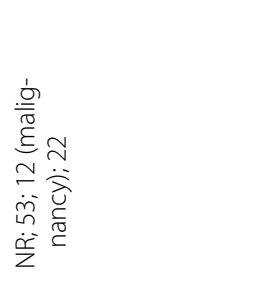 & 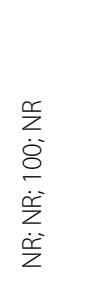 \\
\hline 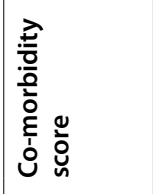 & 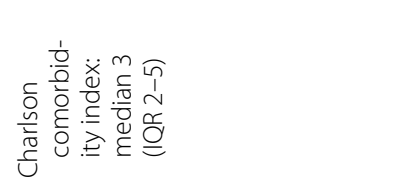 & 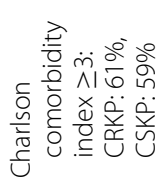 & $\stackrel{\Upsilon}{z}$ & 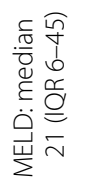 & $\stackrel{\Re}{z}$ & 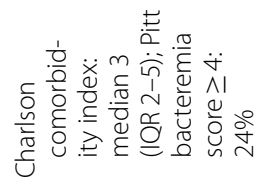 & $\stackrel{\dddot{c}}{z}$ \\
\hline 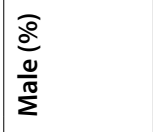 & F & 응 & ટ્ర & $\bar{\infty}$ & $\frac{œ}{z}$ & $\mathscr{f}$ & 岕 \\
\hline 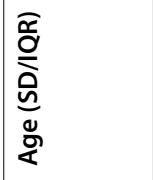 & 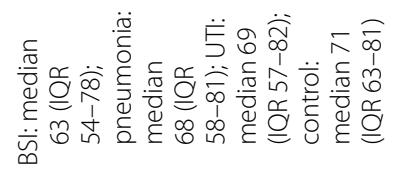 & 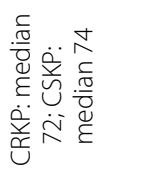 & 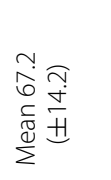 & 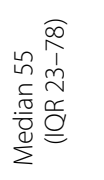 & 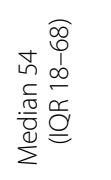 & 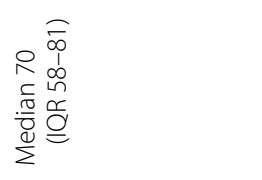 & 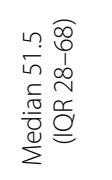 \\
\hline 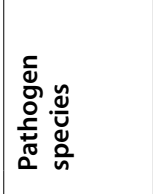 & 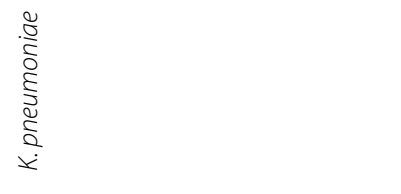 & 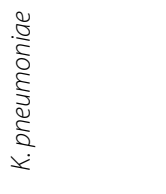 & 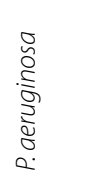 & 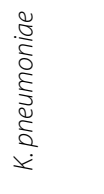 & 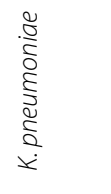 & 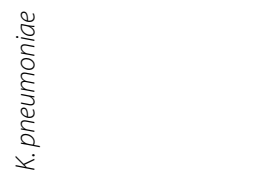 & 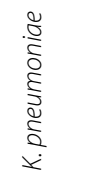 \\
\hline 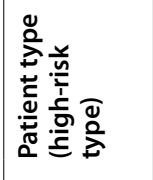 & 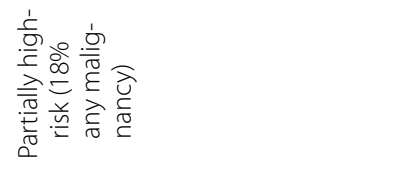 & 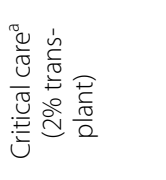 & 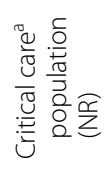 & 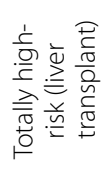 & 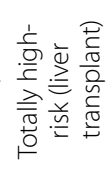 & 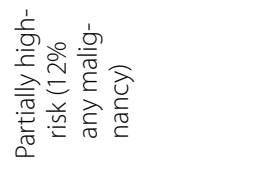 & 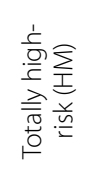 \\
\hline 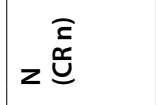 & 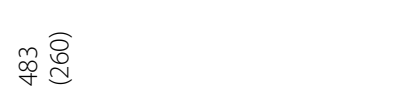 & 吕 & 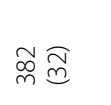 & 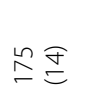 & $\stackrel{\circ}{m} \widehat{\infty}$ & 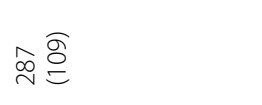 & $\approx \stackrel{\widehat{O}}{\varrho}$ \\
\hline 兽总 & 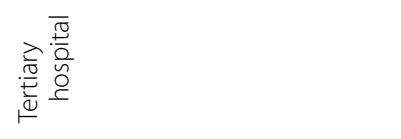 & $\stackrel{\frac{a}{z}}{Z}$ & 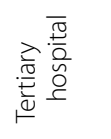 & 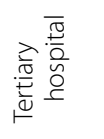 & $\frac{⿱}{z}$ & 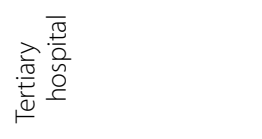 & 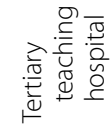 \\
\hline 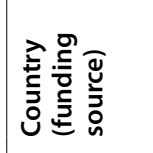 & 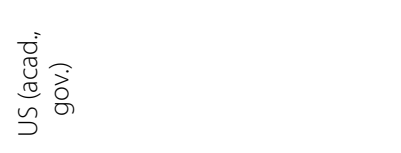 & 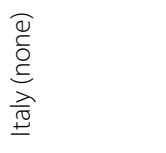 & $\begin{array}{l}\underset{\widetilde{s}}{\sum} \\
\stackrel{心}{J}\end{array}$ & 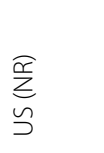 & 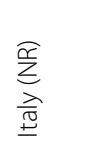 & 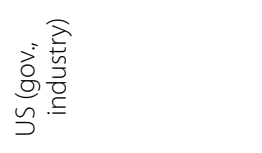 & 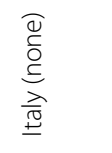 \\
\hline 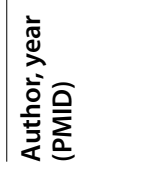 & 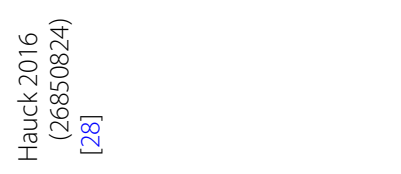 & 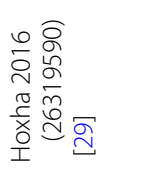 & 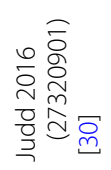 & 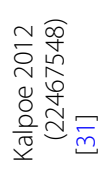 & 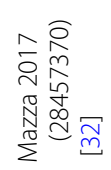 & 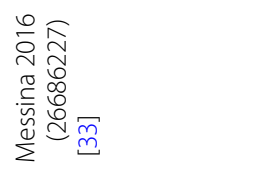 & 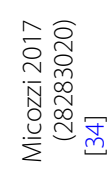 \\
\hline
\end{tabular}




\begin{tabular}{|c|c|c|c|c|c|c|}
\hline 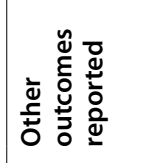 & $\Sigma$ & 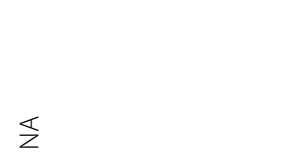 & $\Sigma$ & 岸 & 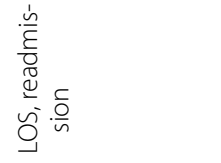 & $\mathbb{z}$ \\
\hline 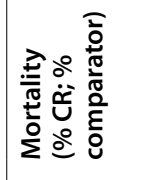 & 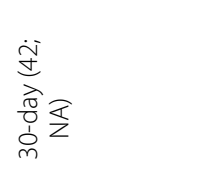 & 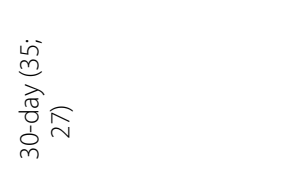 & 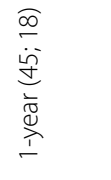 & 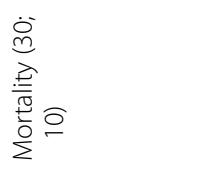 & 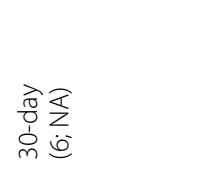 & 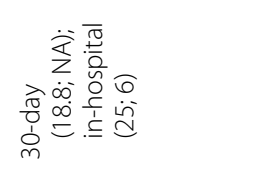 \\
\hline 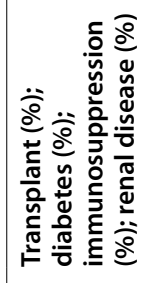 & 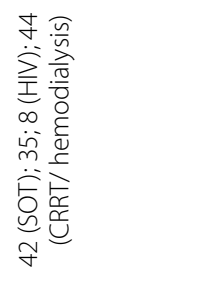 & 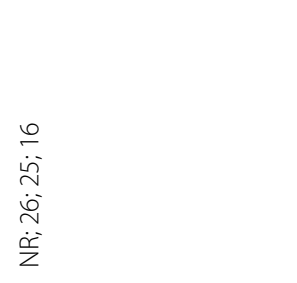 & 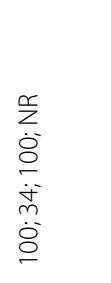 & 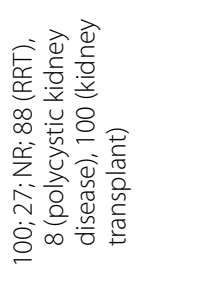 & 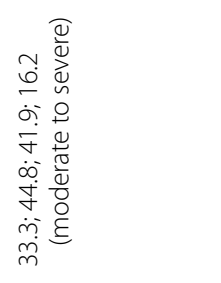 & 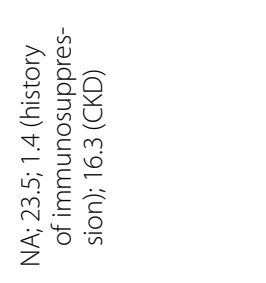 \\
\hline 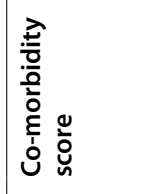 & 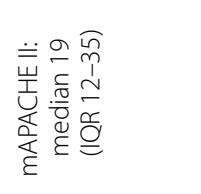 & 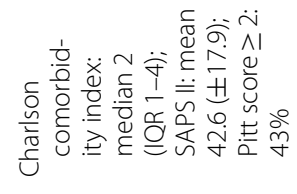 & & $\frac{⿱}{z}$ & 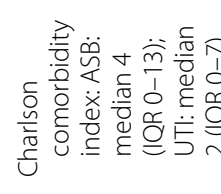 & 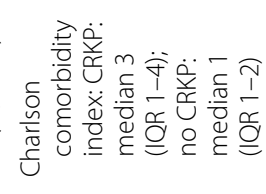 \\
\hline$\frac{\partial}{\frac{\partial}{0}}$ & $\hat{6}$ & ఠิ & $\hat{\sigma}$ & F & $\bar{m}$ & $\begin{array}{l}\sim_{0} \\
\infty \\
0\end{array}$ \\
\hline 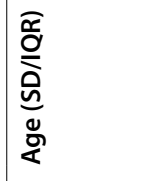 & 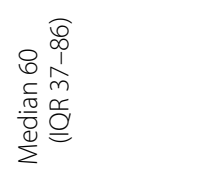 & 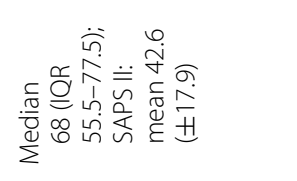 & 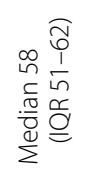 & 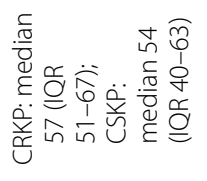 & 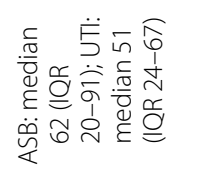 & 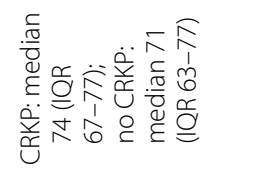 \\
\hline 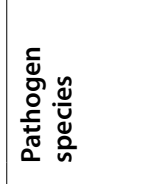 & 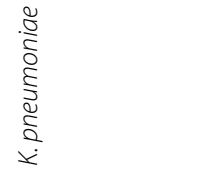 & 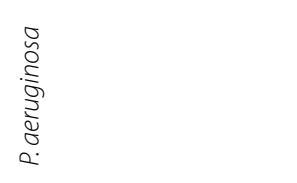 & 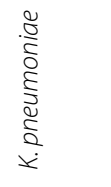 & 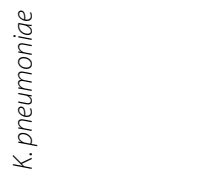 & 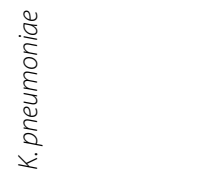 & 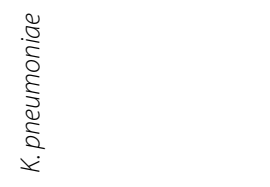 \\
\hline 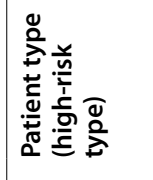 & 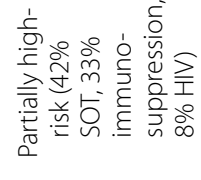 & 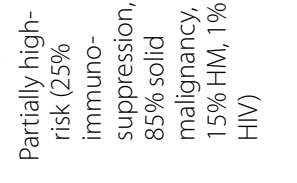 & 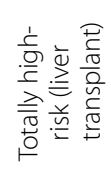 & 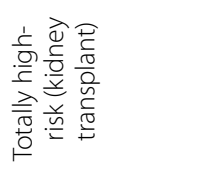 & 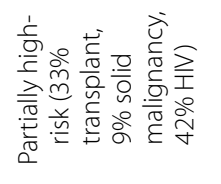 & 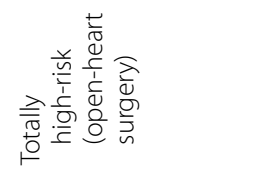 \\
\hline$z$ & $\stackrel{\infty}{\stackrel{o ্}{+}}$ & 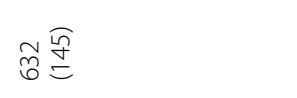 & ১্ৰ & $\stackrel{\circ}{\mathrm{d}}$ & $\stackrel{m}{\underline{m}} \stackrel{\widehat{m}}{=}$ & 命 \\
\hline 总 & 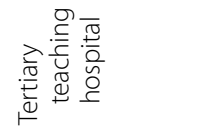 & 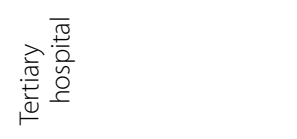 & 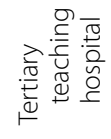 & 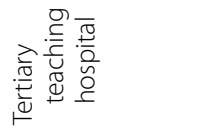 & 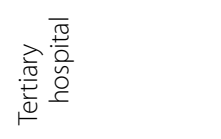 & 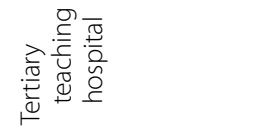 \\
\hline 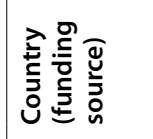 & $\begin{array}{l}\stackrel{\widetilde{c}}{\Sigma} \\
\stackrel{心}{J}\end{array}$ & 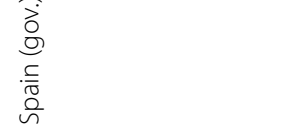 & 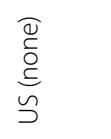 & 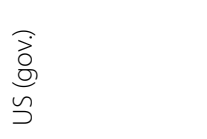 & 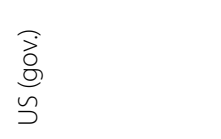 & 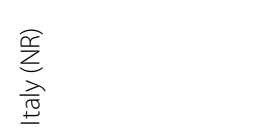 \\
\hline 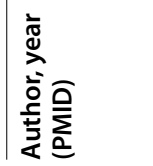 & 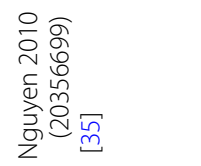 & 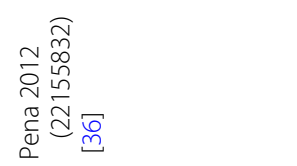 & 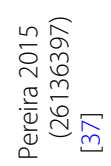 & 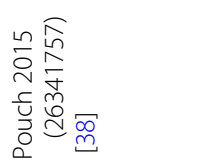 & 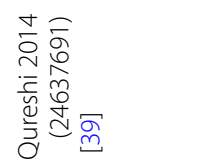 & 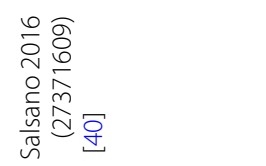 \\
\hline
\end{tabular}




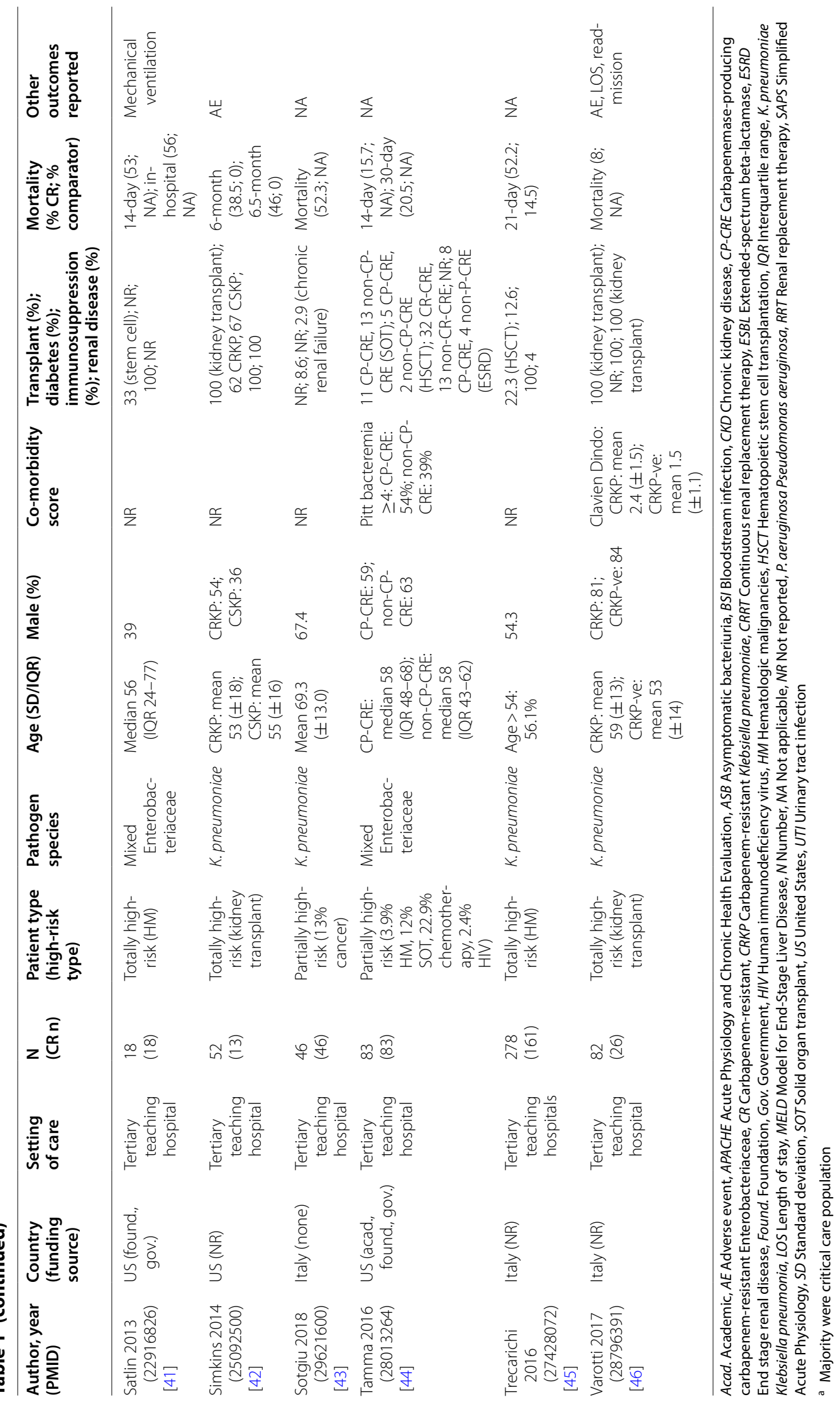




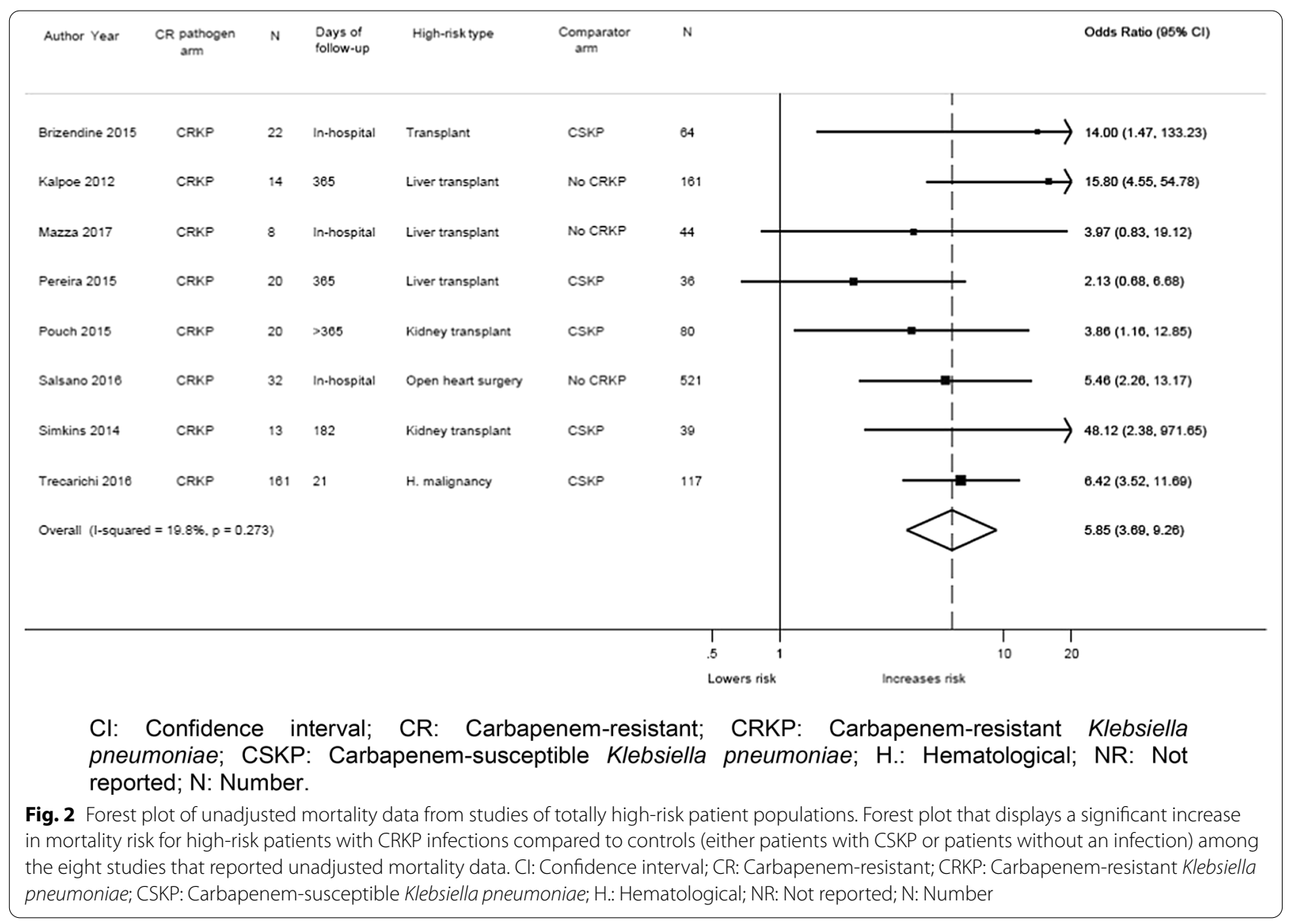

5.07; 95\% CI: 3.38, 7.59; $\mathrm{I}^{2}=0.0 \%$ ). Subgroup analysis by CRKP vs. CSKP (5 studies [13, 37, 38, 42, 45]; summary OR: 5.24; 95\% CI: 2.65, 10.37; $\left.\mathrm{I}^{2}=32.1 \%\right)$, CRKP vs. patients who did not become infected with CRKP (no CR infection) (3 studies [31, 32, 40]; summary OR: 7.02; 95\% CI: 3.33, 14.80; $\mathrm{I}^{2}=18.4 \%$ ), and CRKP vs. no infection (2 studies [32, 37]; summary OR: 24.76; 95\% CI: 4.14, $148.00 ; \mathrm{I}^{2}=71.4 \%$ ) found that there was a significantly higher risk of mortality in patients with CR infections irrespective of the comparator (Table 2). Additional subgroup meta-analysis of unadjusted mortality data at different time points similarly found a significantly higher risk of mortality in patients with $\mathrm{CR}$ infections than in controls: in-hospital/ $\leq 30$-day mortality (4 studies [13, 32, 40, 45]; summary OR: 6.08; 95\% CI: 3.83, 9.66; $\mathrm{I}^{2}=0.0 \%$ ) and longer follow-up (greater than 6 months) (5 studies [31, 37, 38, 40, 42]; summary OR: 6.56; 95\% CI: 2.81, 15.33; $\mathrm{I}^{2}=51.3 \%$ ) (Table 2 ).

Meta-analysis of the five studies that also reported multivariable data [31, 37, 38, 40, 45] (adjusted for various confounders (Additional Table 5)) compared 239 patients with CR infections to 1097 controls and found a significantly increased risk of mortality (summary HR: 4.67; 95\% CI: 2.18, 9.99; $\mathrm{I}^{2}=77.7 \%$ ) (Fig. 3). In sensitivity analysis, excluding one study with an unclear risk of bias [31], the analysis also found a significantly increased risk of mortality (summary HR: 4.62; 95\% CI: $\left.1.87,11.42 ; \mathrm{I}^{2}=83.3 \%\right)$. In sensitivity analysis, excluding one potential outlier study by Salsano et al. [40] (considered as such due to its large CI and exclusion of < 30 day deaths from the 180-day follow-up analysis), the analysis still found a significantly increased mortality among patients with CR infections compared to controls (summary HR: 3.57; 95\% CI: 1.79, 7.14; $\mathrm{I}^{2}=60.4 \%$ ). Studies reported a significantly higher risk of mortality in patients with CR infections irrespective of the comparator. The risk of mortality was also found to be significantly increased in high-risk patients compared with controls in subgroup meta-analysis of four studies reporting adjusted data at longer follow-up periods (> 30 days) (4 studies [31, 37, 38, 40]; summary HR: 6.67; 95\% CI: 3.88, 11.49; $\mathrm{I}^{2}=0.0 \%$ ) (Table 3). Only one study reported adjusted in-hospital/ $\leq 30$-day mortality data [45]; mortality was significantly increased 
Table 2 Meta-analysis results using calculated unadjusted ORs

\begin{tabular}{|c|c|c|}
\hline Analysis for unadjusted mortality data & Studies (N) & Calculated OR (95\% Cl) \\
\hline Totally high-risk patient populations & 8 & $5.85(3.69,9.26) ;\left.\right|^{2}=19.8 \%$ \\
\hline Low risk of bias & 6 & $5.07(3.38,7.59) ; 1^{2}=0.0 \%$ \\
\hline CS infection comparator & 5 & $5.24(2.65,10.37) ;\left.\right|^{2}=32.1 \%$ \\
\hline No CR infection comparator & 3 & $7.02(3.33,14.80) ; 1^{2}=18.4 \%$ \\
\hline No infection comparator & 2 & $24.76(4.14,148.00) ;\left.\right|^{2}=71.4 \%$ \\
\hline |n-hospital/ $\leq 30$-day mortality & 4 & $6.08(3.83,9.66) ; 1^{2}=0.0 \%$ \\
\hline Longer-term (> 30 days) mortality & 5 & $6.56(2.81,15.33) ;\left.\right|^{2}=51.3 \%$ \\
\hline Italy & 3 & $5.86(3.65,9.41) ; 1^{2}=0.0 \%$ \\
\hline USA & 5 & $5.40(2.58,11.30) ;\left.\right|^{2}=34.1 \%$ \\
\hline Partially and totally high-risk patient populations & 14 & $4.13(2.70,6.31) ; 1^{2}=71.5 \%$ \\
\hline Low risk of bias & 9 & $3.94(2.32,6.68) ;\left.\right|^{2}=71.4 \%$ \\
\hline CS infection comparator & 10 & $3.39(2.11,5.45) ; I^{2}=70.2 \%$ \\
\hline No CR infection comparator & 3 & $7.02(3.33,14.80) ;\left.\right|^{2}=18.4 \%$ \\
\hline No infection comparator & 3 & $13.39(4.09,43.87) ;\left.\right|^{2}=75.0 \%$ \\
\hline In-hospital/ $\leq 30$-day mortality & 10 & $3.74(2.37,5.89) ; I^{2}=74.2 \%$ \\
\hline Longer-term (> 30 days) mortality & 5 & $6.56(2.81,15.33) ;\left.\right|^{2}=51.3 \%$ \\
\hline CRKP studies only & 12 & $4.68(3.03,7.23) ;\left.\right|^{2}=61.0 \%$ \\
\hline CRPA studies only & 2 & $2.25(0.84,6.03) ;\left.\right|^{2}=77.6 \%$ \\
\hline Italy & 5 & $4.21(2.17,8.14) ;\left.\right|^{2}=73.3 \%$ \\
\hline USA & 9 & $3.83(2.54,5.76) ;\left.\right|^{2}=28.1 \%$ \\
\hline
\end{tabular}

CI Confidence interval, CRKP Carbapenem-resistant Klebsiella pneumoniae, CRPA Carbapenem-resistant Pseudomonas aeruginosa, CS Carbapenem-susceptible, $N$ Number, OR Odds ratio

with CRKP compared to CSKP (HR: 1.85; 95\% CI: 1.01 , 3.40) (Table 3).

\section{Meta-analysis of mortality data from all studies (totally and partially high-risk patient populations)}

Meta-analysis of 14 studies reporting unadjusted data [13, 22, 27-32, 36-38, 40,42, 45] found a significant increase in the risk of mortality for patients with CR GN infections $(n=1132)$ compared to controls $(n=2949)$ (summary OR: 4.13; 95\% CI: 2.70, 6.31; $\mathrm{I}^{2}=71.5 \%$ ) (Fig. 4). In sensitivity analysis, excluding five studies [22, $27,29,31,42$ ] with high or unclear risk of bias (Additional Table 6), the analysis still found a significantly increased risk of mortality among patients with CR infections compared to controls (summary OR: 3.94; 95\% CI: 2.32, 6.68; $\left.\mathrm{I}^{2}=71.4 \%\right)$. Subgroup analyses by CR vs. CS (10 studies [13, 22, 27, 29, 30, 36-38, 42, 45]; summary OR: 3.39; 95\% CI: $2.11,5.45 ; \mathrm{I}^{2}=70.2 \%$ ), CRKP vs. patients who did not develop CRKP (3 studies [31, 32, 40]; summary OR: 7.02; 95\% CI: 3.33, 14.08; $\mathrm{I}^{2}=18.4 \%$ ), or CRKP vs. no infection (3 studies [28, 32, 37]; summary OR: 13.39; 95\% CI: 4.09, 43.87; $\mathrm{I}^{2}=75.0 \%$ ) all found that there was a significantly higher risk of mortality in patients with $\mathrm{CR}$ infections irrespective of the comparator (Table 2). Additional subgroup meta-analysis of unadjusted mortality data at different time points similarly found a significantly higher risk of mortality in patients with CR infections than in controls: in-hospital/ $\leq 30$-day mortality (10 studies [13, $22,27-30,32,36,40,45]$; summary OR: $3.74 ; 95 \% \mathrm{CI}$ : $2.37,5.89 ; \mathrm{I}^{2}=74.2 \%$ ) and longer follow-up (greater than 30 days) (5 studies [31, 37, 38, 40, 42]; summary OR: 6.56; 95\% CI: 2.81, 15.33; $\mathrm{I}^{2}=51.3 \%$ ) (Table 2). Finally, mortality is significantly increased irrespective of the pathogen type: CRKP studies only (12 studies [13, 22, 27-29, 31, 32, 37, 38, 40, 42, 45]; summary OR: 4.68; 95\% CI: 3.03, $\left.7.23 ; \mathrm{I}^{2}=61.0 \%\right)$ and carbapenem-resistant $P$. aeruginosa (CRPA) studies only (2 studies [30, 36]; summary OR: 2.25; 95\% CI: 0.84, 6.03; $\mathrm{I}^{2}=77.6 \%$ ) (Table 2).

Meta-analyses of three studies including patients that were not categorized as high-risk, but who were treated in a critical care setting (408 patients with CR infections and 561 controls) found an increase in the risk for mortality (3 studies [22, 29, 30]; summary OR: 3.30 ; $95 \%$ CI: $\left.1.52,7.14 ; \mathrm{I}^{2}=71.4 \%\right)$.

Eight studies reported multivariate results (adjusted for various confounders (Additional Table 5)) comparing patients with CR infections $(n=560)$ to controls $(n=1840)$. Meta-analysis of the adjusted data showed a significantly increased risk of mortality for patients with CR GN infections compared to controls (8 studies [27, $28,30,31,37,38,40,45]$; summary HR: 3.38 ; $95 \% \mathrm{CI}$ : 1.93, 5.94; $\mathrm{I}^{2}=76.0 \%$ ) (Fig. 5). This significant increase 


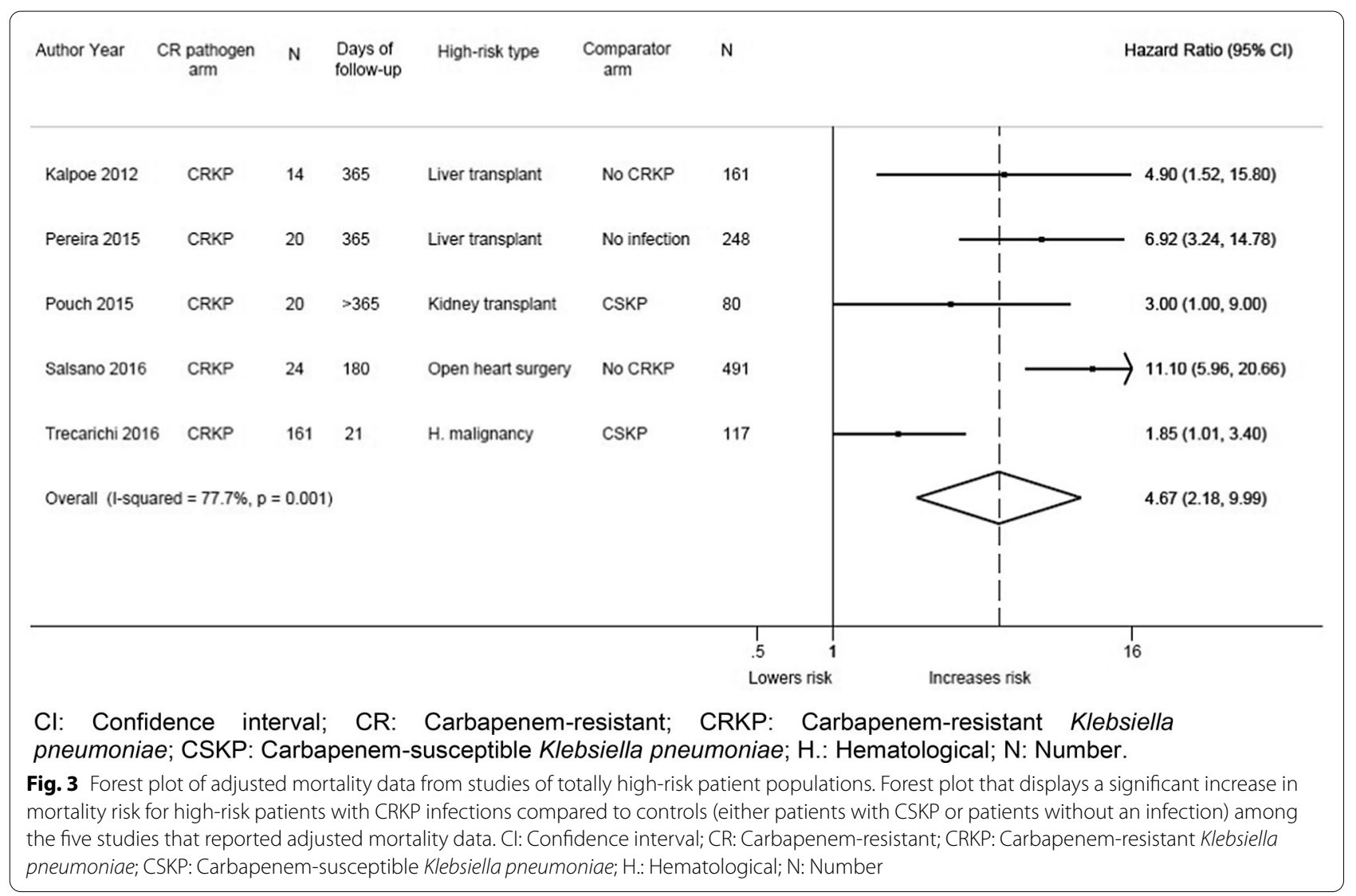

Table 3 Meta-analysis results using reported multivariable adjusted data

\begin{tabular}{|c|c|c|}
\hline Analysis for adjusted mortality data & Studies (N) & Adjusted HR $(95 \% \mathrm{Cl})$ \\
\hline Totally high-risk patient populations & 5 & $4.67(2.18,9.99) ;\left.\right|^{2}=77.7 \%$ \\
\hline Low risk of bias & 4 & $4.62(1.87,11.42) ;\left.\right|^{2}=83.3 \%$ \\
\hline CS infection comparator & 2 & $2.07(1.22,3.53) ; I^{2}=0.0 \%$ \\
\hline No CR infection comparator & 2 & $8.63(4.12,18.08) ; I^{2}=31.6 \%$ \\
\hline No infection comparator & 1 & $6.92(3.24,14.79)$ \\
\hline Longer-term (> 30 days) mortality & 4 & $6.67(3.88,11.49) ;\left.\right|^{2}=0.0 \%$ \\
\hline Italy & 2 & $4.53(0.78,26.20) ;\left.\right|^{2}=93.3 \%$ \\
\hline USA & 3 & $5.19(2.99,9.01) ;\left.\right|^{2}=0.0 \%$ \\
\hline Partially and totally high-risk patient populations & 8 & $3.38(1.93,5.94) ; 1^{2}=76.0 \%$ \\
\hline Low risk of bias & 6 & $3.87(2.10,7.13) ;\left.\right|^{2}=76.9 \%$ \\
\hline CS infection comparator & 4 & $1.93(1.24,2.99) ;\left.\right|^{2}=8.2 \%$ \\
\hline No CR infection comparator & 2 & $8.63(4.12,18.08) ; I^{2}=31.6 \%$ \\
\hline No infection comparator & 2 & $4.08(1.56,10.65) ;\left.\right|^{2}=76.5 \%$ \\
\hline In-hospital/ $\leq 30$-day mortality & 4 & $2.05(1.39,3.02) ;\left.\right|^{2}=16.9 \%$ \\
\hline Longer-term (> 30 days) mortality & 4 & $6.67(3.88,11.49) ; I^{2}=36.6 \%$ \\
\hline CRKP studies only & 7 & $3.45(1.83,6.51) ;\left.\right|^{2}=79.3 \%$ \\
\hline Studies reporting HR & 6 & $4.17(2.23,7.80) ;\left.\right|^{2}=76.6 \%$ \\
\hline Studies reporting OR & 2 & $1.71(0.60,4.82) ;\left.\right|^{2}=60.1 \%$ \\
\hline Italy & 2 & $4.53(0.78,26.20) ;\left.\right|^{2}=93.3 \%$ \\
\hline USA & 6 & $3.02(1.79,5.08) ;\left.\right|^{2}=54.2 \%$ \\
\hline
\end{tabular}




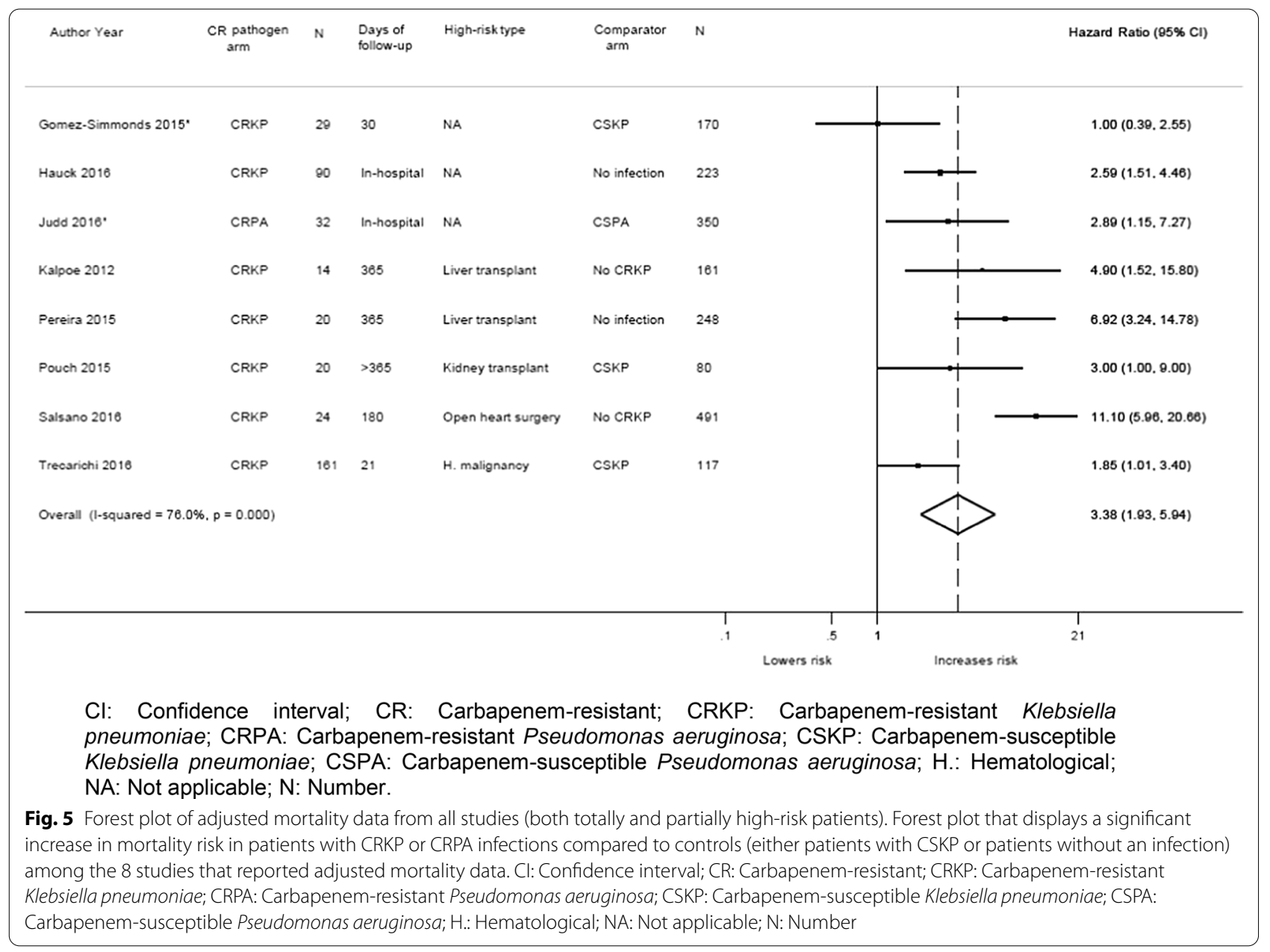

reported 39\% 7-day mortality, 53\% 14-day mortality, and $56 \%$ in-hospital mortality in patients with hematological malignancy and bloodstream infection due to CR Enterobacteriaceae (majority with $K$. pneumoniae). Salsano 2016 [40] reported $18.8 \%$ 30-day mortality in open heart surgery patients with CRKP.

\section{Mechanical ventilation}

Two studies reported mechanical ventilation data [32, 41]. One study [32] reported that all of the liver transplant recipients with CRKP $(n=8)$ required mechanical ventilation during the post-transplant period compared to $64 \%$ of liver transplant recipients infected with microorganisms other than CRKP. In the second study (no comparator [41]), $11 \%$ of the 18 patients with hematologic malignancies and bloodstream infections due to CR Enterobacteriaceae required mechanical ventilation.

\section{Adverse events}

Five studies reported adverse events; these studies all examined transplant recipients with CRKP infection [24,
32, 38, 42, 46] . Three of these studies reported graftrelated outcomes in kidney transplant recipients, comparing CRKP with either CSKP patients or patients who never became CRKP positive during the follow-up period $[38,42,46]$. Only one of these studies reported a significant difference, in delayed graft function ( $42 \%$ vs. $17 \%$, $p=0.03$ ) for patients infected with CRKP versus patients who never became CRKP positive during the follow-up period [46]. This study also reported significantly more acute rejections in patients with CRKP infection versus patients who did not become CRKP positive ( $11 \%$ vs. $0 \%$, $\mathrm{p}=0.03)$. In two other studies, there was no significant difference between patients with CRKP vs. CSKP infection for either graft failure (20\% vs. $16 \%, p=0.73)$ [38] or graft loss ( $15 \%$ vs. $3 \%, p=0.15)$ [42], and no difference in the rate of rejection between CRKP- and CSKP-infected patients (20\% vs. $18 \%, p=0.47)$ [38].

Three studies reported renal adverse events [24, 32, 38]. Mazza 2017 [32] reported a significantly higher use of renal replacement therapy among liver transplant recipients infected with CKRP compared to patients infected 
with micro-organisms other than CRKP (87\% vs. $41 \%$, $p=0.018$ ). Clancy 2013 [24] reported that $29 \%$ of transplant patients infected with CRKP developed antibioticinduced renal failure. The Pouch 2015 study [38] also reported that $20 \%$ of CRKP patients developed nephrotoxicity with antimicrobial therapy, but did not report results for the CSKP arm.

Finally, Varotti 2017 [46] reported significantly more medical complications in patients with CRKP infections than in patients who did not become CRKP positive (92\% vs. $19 \%, p<0.001)$.

\section{Readmission}

Three studies reported readmission [33, 39, 46]. In one study reporting CRKP-infected patients [39], 38\% of those with UTI and $31 \%$ of those with asymptomatic bacteriuria were readmitted within 30 days due to causes unrelated to the CKRP infection. In a second study, 20.2\% of CRKP survivors were readmitted within 90 days and were found to be culture-positive for CRKP upon readmission [33]. The time period for readmission was undefined in the third study, which noted readmission in $81 \%$ of CRKP-infected kidney transplant recipients compared to $21 \%$ of kidney transplant recipients who never became CRKP positive during the follow-up [46].

\section{Cost}

Judd and colleagues [30] compared costs for patients with meropenem-resistant or meropenem-susceptible P. aeruginosa (MRPA vs. MSPA) infections; for patients admitted between 2011 and 2013 the total visit cost was significantly higher $(\mathrm{p}<0.001)$ for MRPA compared to MSPA (median: $\$ 37,331$; range: $\$ 17,141-\$ 77,333$ vs. $\$ 15,995$; range: $\$ 8542-\$ 31,811)$.

\section{Length of stay}

Six studies were identified; five reported median length of stay $[28,30,39,40,46]$ and two reported median length of ICU stay [30, 32]. Three of the six studies included high-risk patients only [32, 40,46] Across studies, the median length of stay was significantly longer for CRKPinfected patients compared to patients with CS infection or no infection (ranging from 10 to 41 days vs. 5.6 to 18 days, respectively), except for a subgroup of patients with UTI in one study [28] that reported no significant difference. For ICU stay specifically, one study among liver transplant recipients found a significantly longer median length of stay for CRKP-infected patients than for patients infected with a micro-organisms other than CRKP (32.5 vs. 19.5 days, $p=0.001$ ), and for patients free from infections (32.5 vs. 5.6 days, $\mathrm{p}=0.001$ ) [32], while another study conducted among inpatients found no significant difference in ICU admissions between MRPA and MSPA infections (12 days vs. 6 days, $p=0.052$ ) [30].

\section{Discussion}

This SLR and meta-analysis of contemporary literature outlines the burden of CR GN infections among highrisk patients. To the best of our knowledge, this is the first review that focuses on high-risk patients specifically. Two prior reviews among non-high-risk hospitalized patients reported two-fold increases in mortality among patients with CR Enterobacteriaceae infections compared to CS Enterobacteriaceae [47, 48]; our analysis found an almost six-fold increase in mortality with CR GN infections in high-risk patients (5-fold increase compared to CS infection or 8-fold increase compared to no infection). The association between CR infection and increased mortality was consistent across studies solely including high-risk patients or studies including partial high-risk populations. Increased mortality was also observed irrespective of the length of follow-up (either short-term or long-term) and for different causative pathogens or underlying resistance mechanisms. In contrast, there was no association between CR GN infections and increased mortality in the three studies that did not include any specific high-risk patient groups but did include a substantial number of patients receiving treatment in an ICU.

All outcomes, with the exception of mortality, were summarized descriptively either due to insufficient numbers of studies or heterogeneity between included studies. A limited number of studies found that CR GN infections increased hospital readmissions and costs and increased the risk for mechanical ventilation. Further, this review identified studies which reported an increased risk for adverse events in high-risk patients with CR GN infections, including nephrotoxicity linked to antimicrobial treatment, and graft failure or acute rejection in solid organ transplant recipients. Considering both the increased mortality and adverse events in high-risk patients, these findings highlight the need for new treatment paradigms and novel treatments with good efficacy and tolerability profiles to achieve more favorable outcomes for patients with high unmet need.

As with any evidence synthesis approach, the limitations of the available data will transfer into limitations of the SLR. First, the studies eligible for inclusion were heterogeneous with respect to the definition of exposure, site of infection, definitions of carbapenem resistance, and types of controls. Studies reporting on $K$. pneumoniae did not address virulence, and consequently, there may have been variation in $K$. pneumoniae virulence among the studies included in the meta-analysis. Furthermore, we were limited by the 
definition of carbapenem resistance as provided by the study authors, so there may also be variation in the carbapenem resistance mechanism represented in the included studies (i.e. some, but not all isolates would have been carbapenemase producers). Most eligible studies did not report on the resistance mechanisms in sufficient detail. Confounding of unadjusted data in observational studies is another well-known potential source of bias. These limitations most likely contributed to the high heterogeneity in some of our meta-analyses results. Any association of an exposure with outcomes in the presence of high heterogeneity may misrepresent the true association, and therefore, the results of these meta-analyses should be interpreted with caution. We attempted to mitigate these issues through subgroup analyses, limiting analyses to similar comparators, and combining multivariate adjusted data in meta-analyses, though we acknowledge that certain limitations will persist despite these steps. Second, for all of the outcomes of interest except mortality, it was not possible to perform meta-analysis i.e., two studies might show statistically significant effects of carbapenem resistance on the outcome, but a third study was not available to permit further exploration through meta-analysis. Finally, our study results may not be generalizable to all geographical regions as studies were included from only certain regions (e.g., North America, Western Europe). Although we aimed to include studies conducted throughout Western Europe, the available studies were only from Spain and Italy, and so our results may not be generalizable to other countries in Western Europe. Despite these limitations, we believe this review addresses an important topic and has identified the relevant contemporary information to further our understanding of the unmet need associated with highrisk patients with $\mathrm{CR} G \mathrm{GN}$ infections.

\section{Conclusions}

This SLR and meta-analysis indicates that CR GN infections among high-risk patients are associated with increased mortality. As carbapenem resistance becomes more widespread, and may yet be further exacerbated through misuse or overuse of existing antimicrobials, understanding the burden of CR GN infections and the patients who are most impacted will be necessary to appropriately allocate resources to target and control these resistant infections. These findings further emphasize the need for robust and data-driven antimicrobial stewardship and infection control measures in hospitals which treat high-risk patients and the continuing need for the development of effective antimicrobials with favorable efficacy and safety profiles for the treatment of CR GN infections.

\section{Supplementary information}

Supplementary information accompanies this paper at https://doi. org/10.1186/s13756-020-00858-8.

Additional file 1: Table S1. Search strategy conducted in OVID MEDLINE ${ }^{\circledR}$ on March 31, 2019. Table S2. Search strategy conducted in Embase ${ }^{\circledR}$ on March 31, 2019. Table S3. Reasons for exclusion of 133 articles during fulltext review. Table S4. Comparator for each outcome by study. Table S5. List of confounders in studies reporting adjusted multivariable analyses. Table S6. Risk of bias.

\section{Abbreviations}

AHRQ: Agency of Healthcare Research and Quality; Cl: Confidence interval; CR: Carbapenem-resistant; CRKP: Carbapenem-resistant Klebsiella pneumoniae; CRPA: Carbapenem-resistant Pseudomonas aeruginosa; CS: Carbapenemsusceptible; CSKP: Carbapenem-susceptible Klebsiella pneumoniae; GN: Gramnegative; HR: Hazard ratio; ICU: Intensive care unit; K. pneumoniae: Klebsiella pneumoniae; MRPA: Multidrug-resistant Pseudomonas aeruginosa; MSPA: Multidrug-susceptible Pseudomonas aeruginosa; OR: Odds ratio; P. aeruginosa: Pseudomonas aeruginosa; RR: Risk ratio; SLR: Systematic literature review; UK: United Kingdom; US: United States; UTI: Urinary tract infection.

\section{Acknowledgements}

None.

\section{Authors' contributions}

E.M. designed research; E.E.A, G.R., and J.C. conducted research; E.E.A and G.R. analyzed data; and E.E.A, G.R., E.M. wrote the paper; E.M. edited the paper; G.R. E.E.A, and J.C. had primary responsibility for final content. All authors read and approved the final manuscript.

\section{Funding}

Funding for this review was provided to Tufts Medical Center by Merck Sharp \& Dohme Corp., a subsidiary of Merck \& Co., Inc., Kenilworth, NJ, USA. The funder played no role in the study search and selection, data synthesis and analysis.

Availability of data and materials

Data supporting the conclusions of this article is available in the Additional Material.

Ethics approval and consent to participate

Not applicable.

Consent for publication

Not applicable.

\section{Competing interests}

E. McCann is an employee of Merck Sharp \& Dohme Corp., a subsidiary of Merck \& Co., Inc., Kenilworth, NJ, USA.

\section{Author details}

${ }^{1}$ Institute for Clinical Research and Health Policy Studies, Center for Clinical Evidence Synthesis, Tufts Medical Center, Boston, MA, USA. ${ }^{2}$ Tufts University School of Medicine, Boston, MA, USA. ${ }^{3}$ Merck \& Co., Inc., Kenilworth, NJ, USA.

Received: 21 July 2020 Accepted: 18 November 2020 Published online: 07 December 2020 


\section{References}

1. Boucher HW, Talbot GH, Bradley JS, Edwards JE, Gilbert D, Rice LB, et al. Bad bugs, no drugs: no ESKAPE! An update from the Infectious Diseases Society of America. Clin Infect Dis. 2009;48:1-12.

2. Peleg AY, Hooper DC. Hospital-acquired infections due to gram-negative bacteria. N Engl J Med. 2010;362:1804-13.

3. Vincent J-L, Rello J, Marshall J, Silva E, Anzueto A, Martin CD, et al. International study of the prevalence and outcomes of infection in intensive care units. JAMA. 2009;302:2323-9.

4. Global Priority List of Antibiotic-Resistant Bacteria to Guide Research, Discovery, and Development of New Antibiotics. 2017. http://www.who. int/medicines/publications/global-priority-list-antibiotic-resistant-bacte ria/en/. Accessed 1 Oct 2019.

5. Spapen H, Jacobs R, Van Gorp V, Troubleyn J, Honoré PM. Renal and neurological side effects of colistin in critically ill patients. Ann Intensive Care. 2011;1:14

6. Gasink LB, Brennan PJ. Isolation precautions for antibiotic-resistant bacteria in healthcare settings. Curr Opin Infect Dis. 2009;22:339-44.

7. Ben-David D, Masarwa S, Navon-Venezia S, Mishali H, Fridental I, Rubinovitch $B$, et al. Carbapenem-resistant Klebsiella pneumoniae in post-acutecare facilities in Israel. Infect Control Hosp Epidemiol. 2011;32:845-53.

8. Magiorakos AP, Burns K, Rodríguez Baño J, Borg M, Daikos G, Dumpis U, et al. Infection prevention and control measures and tools for the prevention of entry of carbapenem-resistant Enterobacteriaceae into healthcare settings: guidance from the European Centre for Disease Prevention and Control. Antimicrob Resist Infect Control. 2017;6:113.

9. Nucleo E, Caltagirone M, Marchetti VM, D'Angelo R, Fogato E, Confalonieri $M$, et al. Colonization of long-term care facility residents in three Italian provinces by multidrug-resistant bacteria. Antimicrob Resist Infect Control. 2018;7:33.

10. Zweigner J, Gastmeier P, Kola A, Klefisch F-R, Schweizer C, Hummel M. A carbapenem-resistant Klebsiella pneumoniae outbreak following bronchoscopy. Am J Infect Control. 2014;42:936-7.

11. Kola A, Piening B, Pape U-F, Veltzke-Schlieker W, Kaase M, Geffers C, et al. An outbreak of carbapenem-resistant OXA-48 - producing Klebsiella pneumonia associated to duodenoscopy. Antimicrob Resist Infect Control. 2015:4:8

12. Potron $A$, Bernabeu $S$, Cuzon G, Pontiès V, Blanchard $H$, Seringe $E$, et al. Analysis of OXA-204 carbapenemase-producing Enterobacteriaceae reveals possible endoscopy-associated transmission, France, 2012 to 2014. Euro Surveill. 2017;22:17-00048.

13. Brizendine KD, Richter SS, Cober ED, van Duin D. Carbapenem-resistant Klebsiella pneumoniae urinary tract infection following solid organ transplantation. Antimicrob Agents Chemother. 2015;59:553-7.

14. Nordmann P, Poirel L. The difficult-to-control spread of carbapenemase producers among Enterobacteriaceae worldwide. Clin Microbiol Infect. 2014;20:821-30.

15. Ruppé É, Woerther P-L, Barbier F. Mechanisms of antimicrobial resistance in gram-negative bacilli. Ann Intensive Care. 2015;5:61.

16. Averbuch D, Tridello G, Hoek J, Mikulska M, Akan H, Yanez San Segundo L, et al. Antimicrobial resistance in gram-negative rods causing bacteremia in hematopoietic stem cell transplant recipients: intercontinental prospective study of the infectious diseases working Party of the European Bone Marrow Transplantation Group. Clin Infect Dis. 2017;65:1819-28.

17. Bassetti M, Carnelutti A, Peghin M. Patient specific risk stratification for antimicrobial resistance and possible treatment strategies in gram-negative bacterial infections. Expert Rev Anti-Infect Ther. 2017;15:55-65.

18. Nour I, Eldegla HE, Nasef N, Shouman B, Abdel-Hady H, Shabaan AE. Risk factors and clinical outcomes for carbapenem-resistant gramnegative late-onset sepsis in a neonatal intensive care unit. J Hosp Infect. 2017;97:52-8

19. Awan AA, Niu J, Pan JS, Erickson KF, Mandayam S, Winkelmayer WC, et al. Trends in the causes of death among kidney transplant recipients in the United States (1996-2014). Am J Nephrol. 2018:48:472-81.

20. Freundlich RE, Maile MD, Sferra JJ, Jewell ES, Kheterpal S, Engoren M. Complications associated with mortality in the National Surgical Quality Improvement Program Database. Anesth Analg. 2018;127:55-62.

21. Rathbone J, Hoffmann T, Glasziou P. Faster title and abstract screening? Evaluating Abstrackr, a semi-automated online screening program for systematic reviewers. Syst Rev. 2015;4:80
22. Alicino C Giacobbe DR Orsi A Tassinari F Trucchi C Sarteschi G et al. Trends in the annual incidence of carbapenem-resistant Klebsiella pneumoniae bloodstream infections: a 8-year retrospective study in a large teaching hospital in northern Italy. BMC Infect Dis. 2015;15:415.

23. Capone A, Giannella M, Fortini D, Giordano A, Meledandri M, Ballardini M, et al. High rate of colistin resistance among patients with carbapenemresistant Klebsiella pneumoniae infection accounts for an excess of mortality. Clin Microbiol Infect. 2013;19:E23-30.

24. Clancy CJ, Chen L, Shields RK, Zhao Y, Cheng S, Chavda KD, et al. Epidemiology and molecular characterization of bacteremia due to carbapenemresistant Klebsiella pneumoniae in transplant recipients. Am J Transplant. 2013:13:2619-33

25. Cristina ML, Alicino C, Sartini M, Faccio V, Spagnolo AM, Bono VD, et al. Epidemiology, management, and outcome of carbapenem-resistant Klebsiella pneumoniae bloodstream infections in hospitals within the same endemic metropolitan area. J Infect Public Health. 2018:11:171-7.

26. Giannella M, Trecarichi EM, Giacobbe DR, De Rosa FG, Bassetti M, Bartoloni $\mathrm{A}$, et al. Effect of combination therapy containing a highdose carbapenem on mortality in patients with carbapenem-resistant Klebsiella pneumoniae bloodstream infection. Int J Antimicrob Agents. 2018;51:244-8.

27. Gomez-Simmonds A, Greenman M, Sullivan SB, Tanner JP, Sowash MG, Whittier $S$, et al. Population structure of Klebsiella pneumoniae causing bloodstream infections at a new York City tertiary care hospital: diversification of multidrug-resistant isolates. J Clin Microbiol. 2015;53:2060-7.

28. Hauck C, Cober E, Richter SS, Perez F, Salata RA, Kalayjian RC, et al. Spectrum of excess mortality due to carbapenem-resistant Klebsiella pneumoniae infections. Clin Microbiol Infect. 2016;22:513-9.

29. Hoxha A, Kärki T, Giambi C, Montano C, Sisto A, Bella A, et al. Attributable mortality of carbapenem-resistant Klebsiella pneumoniae infections in a prospective matched cohort study in Italy, 2012-2013. J Hosp Infect. 2016;92:61-6.

30. Judd WR, Ratliff PD, Hickson RP, Stephens DM, Kennedy CA. Clinical and economic impact of meropenem resistance in Pseudomonas aeruginosainfected patients. Am J Infect Control. 2016:44:1275-9.

31. Kalpoe JS, Sonnenberg E, Factor SH, del Rio Martin J, Schiano T, Patel G, et al. Mortality associated with carbapenem-resistant Klebsiella pneumoniae infections in liver transplant recipients. Liver Transpl. 2012;18:468-74.

32. Mazza E, Prosperi M, Panzeri MF, Limuti R, Nichelatti M, De Gasperi A. Carbapenem-resistant Klebsiella Pneumoniae infections early after liver transplantation: a single-center experience. Transplant Proc. 2017:49:677-81.

33. Messina JA, Cober E, Richter SS, Perez F, Salata RA, Kalayjian RC, et al. Hospital readmissions in patients with Carbapenem-resistant Klebsiella pneumoniae. Infect Control Hosp Epidemiol. 2016;37:281-8.

34. Micozzi A, Gentile G, Minotti C, Cartoni C, Capria S, Ballarò D, et al. Carbapenem-resistant Klebsiella pneumoniae in high-risk haematological patients: factors favouring spread, risk factors and outcome of carbapenem-resistant Klebsiella pneumoniae bacteremias. BMC Infect Dis. 2017;17:203.

35. Nguyen M, Eschenauer GA, Bryan M, O'Neil K, Furuya EY, Della-Latta P, et al. Carbapenem-resistant Klebsiella pneumoniae bacteremia: factors correlated with clinical and microbiologic outcomes. Diagn Microbiol Infect Dis. 2010;67:180-4

36. Peña C, Suarez C, Gozalo M, Murillas J, Almirante B, Pomar V, et al. Prospective multicenter study of the impact of carbapenem resistance on mortality in Pseudomonas aeruginosa bloodstream infections. Antimicrob Agents Chemother. 2012;56:1265-72

37. Pereira MR, Scully BF, Pouch SM, Uhlemann A-C, Goudie S, Emond JE, et al. Risk factors and outcomes of carbapenem-resistant Klebsiella pneumoniae infections in liver transplant recipients. Liver Transpl. 2015;21:1511-9.

38. Pouch SM, Kubin CJ, Satlin MJ, Tsapepas DS, Lee JR, Dube G, et al. Epidemiology and outcomes of carbapenem-resistant Klebsiella pneumoniae bacteriuria in kidney transplant recipients. Transpl Infect Dis. 2015;17:800-9.

39. Qureshi ZA, Syed A, Clarke LG, Doi Y, Shields RK. Epidemiology and clinical outcomes of patients with carbapenem-resistant Klebsiella pneumoniae bacteriuria. Antimicrob Agents Chemother. 2014;58:3100-4.

40. Salsano A, Giacobbe DR, Sportelli E, Olivieri GM, Brega C, Di Biase C, et al. Risk factors for infections due to carbapenem-resistant Klebsiella 
pneumoniae after open heart surgery. Interact Cardiovasc Thorac Surg. 2016;23:762-8.

41. Satlin MJ, Calfee DP, Chen L, Fauntleroy KA, Wilson SJ, Jenkins SG, et al. Emergence of carbapenem-resistant Enterobacteriaceae as causes of bloodstream infections in patients with hematologic malignancies. Leuk Lymphoma. 2013;54:799-806.

42. Simkins J, Muggia V, Cohen HW, Minamoto GY. Carbapenem-resistant Klebsiella pneumoniae infections in kidney transplant recipients: a casecontrol study. Transpl Infect Dis. 2014:16:775-82.

43. Sotgiu G, Are BM, Pesapane L, Palmieri A, Muresu N, Cossu A, et al. Nosocomial transmission of carbapenem-resistant Klebsiella pneumoniae in an Italian university hospital: a molecular epidemiological study. J Hosp Infect. 2018;99:413-8.

44. Tamma PD, Goodman KE, Harris AD, Tekle T, Roberts A, Taiwo A, et al. Comparing the outcomes of patients with Carbapenemase-producing and non-Carbapenemase-producing Carbapenem-resistant Enterobacteriaceae bacteremia. Clin Infect Dis. 2017;64:257-64.

45. Trecarichi EM, Pagano L, Martino B, Candoni A, Di Blasi R, Nadali G, et al. Bloodstream infections caused by Klebsiella pneumoniae in onco-hematological patients: clinical impact of carbapenem resistance in a multicentre prospective survey. Am J Hematol. 2016;91:1076-81.

46. Varotti G, Dodi F, Terulla A, Santori G, Mariottini G, Bertocchi M, et al. Impact of carbapenem-resistant Klebsiella pneumoniae (CR-KP) infections in kidney transplantation. Transpl Infect Dis. 2017;19:e12757.

47. Martin A, Fahrbach K, Zhao Q, Lodise T. Association Between Carbapenem Resistance and Mortality Among Adult, Hospitalized Patients With Serious Infections Due to Enterobacteriaceae: Results of a Systematic Literature Review and Meta-analysis. Open Forum Infect Dis. 2018:5:ofy150.

48. Cosgrove SE, Carmeli Y. The impact of antimicrobial resistance on health and economic outcomes. Clin Infect Dis. 2003:36:1433-7.

\section{Publisher's Note}

Springer Nature remains neutral with regard to jurisdictional claims in published maps and institutional affiliations.
Ready to submit your research? Choose BMC and benefit from:

- fast, convenient online submission

- thorough peer review by experienced researchers in your field

- rapid publication on acceptance

- support for research data, including large and complex data types

- gold Open Access which fosters wider collaboration and increased citations

- maximum visibility for your research: over $100 \mathrm{M}$ website views per year

At BMC, research is always in progress.

Learn more biomedcentral.com/submissions 